\title{
NARRATIVAS TRANSMEDIALES: TEORÍA, HISTORICIDAD DE LOS MEDIOS COMPARADOS Y CLOSE READING
}

\author{
Domingo SÁNCHEZ-MESA \\ Universidad de Granada \\ dsanchez@ugr.es
}

proximándonos ya al tramo final de la segunda década del siglo XXI, el sintagma —en
inglés - transmedia storytelling, traducido a menudo — no sin necesidad de matices - por
narrativa transmedial, empieza a ser bastante familiar para el investigador en estudios literarios y comparatistas, como lo es también para los estudiosos de los nuevos medios de comunicación digital interactiva o para los expertos en marketing integrado en la producción y comunicación audiovisual. La primera sistematización teórica del concepto ${ }^{1}$ se debe a Henry Jenkins y su archicitado artículo en Technology Review (2003). En una formulación posterior del mismo, el antes profesor del MIT, ahora catedrático de la Anneberg School of Communication de la USC, lo definía así:

A process where integral elements of a fiction get dispersed sistematically across multiple delivery channels for the purpose of creating a unified and coordinated entertainment experience. Ideally, each medium makes its own unique contribution to the unfolding of the story (Jenkins, 2009).

En poco más de una década, las narrativas transmediales y el transmedia storytelling ${ }^{2}$ se han erigido en uno de esos fenómenos o tendencias transversales que, en un contexto de inédita inestabilidad de la red sociotécnica (Scolari 2016, apud Lévy), ha pasado a ocupar posiciones centrales en los discursos tanto académicos como industriales (cuyas importantes diferencias señalara también recientemente M.-L. Ryan, 2016). En el número anterior de esta misma revista ensayamos una propuesta de clarificación teórica donde, en contrapunto con el concepto de intermedialidad —al que consideramos condición de posibilidad de la nueva transmedialidad- distinguíamos, por un lado, la transmedialidad en tanto tendencia a que determinados temas, mitos, argumentos, personajes o mundos de ficción aparezcan en distintos medios. Esta versión histórica o predigital de la transmedialidad nos llevaría a una invitación a matizar la radical novedad de las formas de narrativa distribuida en distintos

\footnotetext{
${ }^{1}$ Fue Marsha Kinder quien, en 1991, habló por primera vez de «intertextualidad transmedia» en Playing with Power in Movies, Television and Video Games: from Muppet Babies to Teenage Mutant Ninja Turtles. Berkeley, University of California Press.

${ }^{2}$ Para ver las diferencias que conviene establecer entre ambos sintagmas véase Sánchez-Mesa/Baetens (2017).
} 
medios o plataformas —el transmedia storytelling de Jenkins- que constituiría, por otro lado, el segundo de los sentidos de la transmedialidad considerados. En dicho artículo, además de ensayar una crítica de la reducción de los procesos de transmedialización a ese segundo sentido, coincidente con el desarrollo de las franquicias crossmediales hacia un tipo de producción coordinada y distribuida, narrativamente hablando, y abierta y permeable a nuevos tipos de participación agente por parte de audiencias más proactivas, tratábamos también de ilustrar, con una serie de casos heterogéneos en su filiación mediática, cuál sería la posición de la literatura en este entramado mutante (Sánchez-Mesa y Baetens, 2017).

Este dossier que aparece ahora en la revista Tropelías — que sigue al que publicamos en el número 18 de Artnodes (Sánchez-Mesa, Alberich y Rosendo, coords., 2016) - cuenta, por una parte, con artículos escritos por cinco autores que, proviniendo del dominio de los estudios teórico-literarios y comparatistas, proyectan sus perspectivas teóricas y metodologías de análisis al ámbito de la transmedialidad y los nuevos medios audiovisuales. Por otra parte, hay otros cuatro investigadores que desarrollan su actividad en el dominio académico de los estudios de comunicación en medios audiovisuales, cuyo discurso está fertilizado por la estética, la literatura comparada y los estudios culturales. Este es el espacio intersticial o dialógico que ha venido ocupando el proyecto i+d Nar$\underline{\text { Trans }}^{3}$ desde el inicio de su andadura (2014) y que tratamos de consolidar con cada encuentro académico, con cada publicación científica. En esta ocasión son tres los ejes que vehiculan esta propuesta:

$1^{\circ}$ TEORÍA: expandir y profundizar en nuestra participación en el debate teórico sobre qué son las narrativas transmediales, tratando de dar respuestas precisas a las posiciones polarizadas que defienden bien la radical novedad del transmedia storytelling, bien que estas formas de producción, creación y consumo cultural no suponen ninguna novedad en las dinámicas crossmedia de las industrias culturales y del entretenimiento. En este dossier exploramos dominios extendidos de la estética, de la literatura y la narratología comparadas, de la teoría literaria y de los new media, de los estudios culturales y del marketing en la producción transmedial.

$2^{\circ}$ HISTORICIDAD de los MEDIOS COMPARADOS: la literatura comparada y los estudios de medios comparados — no debemos olvidar que tal era el nombre del departamento que coordinaba Henry Jenkins en el MIT en el momento de publicar Convergence Culture, 2006- nos invitan a elucidar las distintas formas y funciones de la intermedialidad en la historia de los medios de creación y comunicación artística y cultural. Y dicho espacio de elucubración viene informado por una conciencia clara de la historicidad de estas prácticas culturales que denominamos transmediales y el posible paradigma comunicativo del que forma parte su emergencia. Por ello no pocos de los textos recogidos aquí o bien miran en el pasado, analizando precedentes o formas pretéritas de transmedialización, con un especial protagonismo de la adaptación, o bien asumen una mirada diacrónica, atenta a la evolución en el tiempo del caso de estudio o concepto que se esté tratando.

\footnotetext{
${ }^{3}$ Se trata del proyecto del Ministerio de Industria, Economía y Competitividad, «Narrativas Transmediales: Nuevos Modos de Ficción Audiovisual, Comunicación Periodística y Performance en la Era Digital», con n. ref. CSO2013-47288-P.
} 


\section{Tropelías. Revista de Teoría de la Literatura y Literatura Comparada, 28 (2017) 3 Narrativas transmediales: teoría, historicidad de los medios comparados y close reading}

$3^{\text {a }}$ CLOSE READING: ya en 2003 Jan Baetens y Jan van Looy llamaron nuestra atención reivindicando la necesidad de recuperar, actualizándolo —en clave cultural—, el método de lectura estrecha, heredado de la tradición crítico literaria formalista, para el caso de las textualidades digitales y, en concreto, de la literatura electrónica. Esta estrategia, aparentemente incompatible con las grandes dimensiones que adquieren «los universos transmediales» y la distribución multiplataforma de los relatos expandidos, seguirá siendo una metodología de análisis valiosa en cuanto se entienda que la especificidad material discursiva de cada medio implicado en dicha red integrada de montaje narrativo, es crucial para su propia eficacia tanto pragmática como estética 4 .

Con esa tríada de principios rectores, revisemos aquí brevemente las propuestas básicas del conjunto de textos reunidos en este dossier.

Actualizando una primera incursión en la estética de los medios digitales (Alberich 2002) el texto que ahora coautoriza Jordi Alberich-Pascual con Francisco J. Gómez — «Tiento para una estética transmedia. Vectores estéticos en la creación, producción, uso y consumo de narrativas transmediales»- abre el dossier explorando la oportunidad y posibilidades de una estética de las narrativas transmediales, a modo de aproximación - «tiento» en sentido musical - a la especificidad de una estética propiamente transmedia. Con sistematicidad muy clarificadora, los autores recorren dichos vectores ofreciendo una radiografía fundamentada en los siguiente conceptos o cualidades: (a) la opticidad transmedia: proyectando a Riegl y doblando la integración estética del mundo representado operado por la técnica plástica de la perspectiva renacentista sobre la nueva integración transmedial de las distintas obras y piezas en las que se distribuyen estas nuevas narrativas; (b) la naturaleza procesual: al margen de jerarquías autoriales de tipo tradicional y de la obra como producto acabado, la creación en sí misma es abordada como un proceso; (c) la transflâneurie: la «libertad de movimiento» de los usuarios en mundos creados de forma «abierta», sin límites fijos y estables. El vagabundeo en experiencias fuertemente espaciales - ya sea físico o metafórico dicho espacio—, dentro y a través del «espectáculo del momento»; (d) la coautoría: a través de la participación en una performatividad creativa por parte de los usuarios. Las obras son ya máquinas de posibilidades narrativas - lo que Espen Aarseth llamaría «cibertextos»— y el destinatario tradicional recibe una coautoría «derivada», siendo sus potenciales respuestas e implicación, de hecho, la clave constructiva de la escritura y diseño de los sistemas transmediales; (e) una estética expandida: desterritorializada, desligada de lugares codificados institucionalmente como estéticos, nomadismo del no-lugar asociado a la virtualidad misma.

En suma, se trata de una apuesta estimulante por la reacentuación y recuperación del paradigma de la estética para, desde el bagaje teórico de sus dos siglos y medio de historia y su reciente amplificación en el dominio de los nuevos medios, seguir reflexionando sobre la comunicación artística, en este caso sobre la combinación de los aspectos sensibles, experienciales, técnicos y sociohistóricos de las prácticas culturales transmediales.

\footnotetext{
${ }^{4}$ Este énfasis en la materialidad específica de cada medio, principio básico en el proyecto de investigación Nar-Trans, viene corroborado por el volumen, editado por la misma Marsha Kinder y Tara McPherson, Transmedia Frictions. The Digital, the Arts and the Humanities (2014).
} 
El artículo de Javier Hernández Ruiz — «Diseñando una recepción participativa para universos transmedia: roles y desafíos»- supone una valiosa aportación dado el perfil híbrido de su autor, creador audiovisual con experiencia en la escritura y diseño de producción de uno de los universos transmediales independientes más complejos y ambiciosos de cuantos se han alumbrado en España, Plot28 (vid. Hernández 2015), y por otro su condición de investigador académico especialista en producción y realización transmedial. Es un texto, por consiguiente, útil tanto para los interesados en el debate teórico sobre la transmedialidad y el transmedia storytelling como para quienes se disponen a afrontar la realización de uno de estos complejos productos. Hernández ubica su discurso en las dinámicas de marketing y en la prioridad de la definición de necesidades o perfiles de los prosumidores o audiencias de los productos transmedia. ¿Cuál es el público objetivo del transmedia storyteller?, ¿cómo se plantea el diseño de las producciones transmediales en función de las narrativas inmersivas?, en definitiva, «¿cómo fomentar la prosumición de un universo transmedia?». Robert Pratten llamaba la atención sobre la importancia de que el marketing sea parte, casi inconscientemente para el usuario, de la experiencia transmedial (2015). Hernández desgrana con precisión las estrategias, pasos y requisitos de los estudios del perfil de los destinatarios de estas producciones - y sus campañastransmediales. Desde la apuesta por el potencial democratizador de este nuevo modo de consumo de contenidos, se abordan claves como la «escalabilidad de los procesos», las fases del proceso de «engagement» -implicación comprometida del prosumidor en la experiencia transmedial- y los principios y prácticas que caracterizan la «cultura participativa»_crowdsourcing, CGU, evolución del fandom, interactividad y nuevo perfil de prosumidores-. Con una rica ilustración en casos y ejemplos destacados de creaciones y producciones transmediales —donde las estrategias de ludificación y los ARGs van ganando terreno-, este artículo reivindica un regreso a la centralidad del relato en la teorización del TS, tras el imperio del «contenido». Hernández tiene la experiencia para poder discriminar y diagnosticar las claves del fracaso o el éxito de determinadas producciones transmediales y por tanto sienta las bases, sólidamente contrastadas con la bibliografía fundamental del campo, de un posible método crítico de las producciones transmedia.

José Manuel Ruiz Martínez repasa en «Hannibal Lecter como caso de personaje transmedia: un estudio de caso» la trayectoria de crecimiento transmedial de uno de los últimos mitos de la literatura y el cine del subgénero de serial killers, Hannibal Lecter, el personaje creado por Thomas Harris en sus novelas. Desde el carácter popular de la ficción que le alumbra, a su establecimiento como personaje «franquicia» por Dino de Laurentiis, sobre todo a partir de Red Dragon (B. Ratner, 2002), pasando por la función de la adaptación en las expansiones transmediales, el trabajo de Ruiz Martínez demuestra con sagacidad los resortes e hitos que señalan la emergencia de un personaje transmedial, confirmando la centralidad de la categoría del personaje transficcional en la construcción del TS para explicar la especificidad de ciertos procesos de transmedialización en el nuevo horizonte de las narrativas transmediales (Rosendo 2016, Freeman 2017). Especialmente original es la función que el autor concede a los paratextos en el ligamento de las piezas que van conformando el edificio de 
la franquicia de un mundo transmedial «a posteriori» o construido sobre la marcha a medida que la producción toma conciencia de las posibilidades comerciales y narrativas de una auténtica distribución transmedial de los contenidos del universo diegético. La lectura de Ruiz Martínez del corpus de piezas del mundo transmedial construido en torno al personaje de Hannibal Lecter — novelas, películas, serie de televisión - nos proporciona una provocadora interpretación final apelando a la importancia de la espacialidad y de la memoria en la construcción de los relatos transmediales.

Mario de la Torre, cineasta documentalista responsable de la realización de las piezas audiovisuales de uno de los proyectos de activismo audiovisual transmedial más interesantes del panorama español, La primavera rosa (2013- ), toma el testigo de Marie-Laure Ryan —autoridad de referencia para una narratología ampliada y consciente de la especificidad de los nuevos medios: «I find transmedia to be a very appropriate mode of presentation for non-fictional projects» (2016: 2)—, para reivindicar que el relato transmedial «pueda tener su base en lo factual, siendo esto igualmente legítimo que su aplicación a partir de mundos ficcionales». Y lo hace atreviéndose a explorar una de las categorías más complejas de «transmedializar» desde el punto de vista teórico, como es la del «narrador»y su contraparte en la enunciación, el «narratario», al mismo tiempo que problematiza la noción de «mundo», tal y como se desarrolla en la teoría del transmedia storytelling —basada sobre todo en la teoría de los mundos ficcionales o imaginarios (Wolf, 2012) - . Por otro lado, es evidente que el documental interactivo, también denominado webdoc o i-doc es uno de los géneros o modos de producción de narrativas transmediales más en boga en la creación audiovisual interactiva. Según la definición - provisional - que ofreció Arnau Gifreu, podríamos referirnos al documental interactivo como «interactive applications, on or off-line, made with the intention of representing reality with its own mechanisms that we can call modes of browsing or interaction, relative to the level of participation allowed» (Gifreu, 2011). El artículo completa estas disquisiciones de orden teórico con la descripción y análisis de dos producciones documentales transmediales, el propio proyecto de activismo documental transmedia de La primavera rosa, construido sobre la base de tres documentales, expandidos a través de sendos documentarybooks, la web, exposiciones fotográficas, performances, y Cromosoma Cinco (Lisa Pram y María Ripoll, Lab Interactivos RTVE, 2013), donde cine documental, fotografía y literatura testimonial se complementan y articulan entre sí para ofrecer otro relato que trata de registrar una faceta de la realidad para mover conciencias, en este caso, en torno a una de las llamadas «enfermedades raras».

Precisamente la memoria — a la que nos referíamos más arriba-, en este caso la memoria colectiva histórica, es el pivote sobre el que bascula el texto de Antonio Alías «Exponer a los vencidos: memoria, transmedia y emancipación». En él, su autor traza una concienzuda revisión del hiato existente entre la formulación jurídica y la proyección legal y política del discurso y el derecho a la memoria - contextualizado en el caso español—, respecto al pensamiento crítico entendido como «discurso de la memoria». En la base de dicho pensamiento, se repasa la base filosófica de este discurso en aquella «nueva teoría del conocimiento» y «orden político desde el materialismo histórico basados 
en la experiencia sufriente del sujeto» que, de la mano fundamentalmente de Walter Benjamin y sus Tesis sobre el concepto de historia, pero también del principio del deber de la memoria de Theodor L. W. Adorno, se recupera recientemente con un renovado horizonte de materialización de un «conocimiento justo de los que quedaron fuera de la historia: los vencidos». La estrategia básica de los herederos de este pensamiento consistirá en una «visibilización de los oprimidos», o de «los sin nombre», en expresión de Reyes Mate, estrategia cuyas posibilidades y contradicciones en el mundo de la imagen y los medios ha recorrido otro autor importante para este artículo, el francés Didi-Huberman, quien con su apuesta por un modo narrativo «otro» de inspiración vanguardista, reacentúa el concepto de montaje documental para evitar la reificación de lo representado, en este caso, el sufrimiento de un pueblo vencido. Ese sentido «constructivo» y «materialista» formal de la representación de la historia finalmente atraviesa el proyecto transmedial que describe y valora Alías, Vencidxs, una narrativa transmedial impulsada por numerosos colectivos asociados a la memoria histórica y críticos con la Ley española promulgada en $2007^{5}$. Apoyado en el crowdfunding o creación colaborativa, este proyecto se configura como un experimento y experiencia de activismo transmedial (Srivastava, 2014) de gran potencial sociocultural, que ilustra muy bien, volviendo finalmente a Benjamin, la idea de la técnica como emancipación —en este caso los nuevos medios audiovisuales—, también presente en su seminal ensayo sobre La obra de arte en la era de su reproductibilidad técnica.

Entre los aspectos o dimensiones críticas - sujetas a una intensa metamorfosis - de la creación y la comunicación cultural propias de las artes y los entornos digitales interactivos, sin duda están tanto la cuestión de la autoría, como de la particular permeabilidad y borradura de las fronteras entre la ficción y los hechos, o en términos del mismo Genette, entre el discurso ficcional y discurso factual. En este contexto, el trabajo de Sarai Adarve — «La comedia autoficcional negativa: Louie y El fin de la comedia. Un apunte intermedial»- explora las migraciones del discurso autoficcional hacia el ámbito de las series de televisión, en el caso de Louie (FX 2010-) y El fin de la comedia (Comedy Central 2014-), casos ambos que recorren vías paralelas de deconstrucción del modelo de identidad subjetiva moderno - en torno a los humoristas protagonistas de ambas series, Louis C.K. e Ignatius Farray-, haciendo un uso de la parodia que la autora relaciona con el concepto de posthumor — del crítico cinematográfico Jordi Costa—, claramente identificable dentro de la estética postmoderna.

Por último, el trabajo que presentamos Jan Baetens y Domingo Sánchez-Mesa — «La Ventana Indiscreta de Alfred Hitchcock como Cineromanzo. La adaptación como transmedialización»ensaya un ejercicio de arqueología de los procesos de transmedialización, con la práctica de la adaptación en el centro del análisis, en este caso a propósito de un medio que podemos considera «muerto», esto es el cine-fotonovela o, según la denominación italiana, cineromanzo. Decimos «muerto» porque se trata de un medio cultivado durante los años cincuenta y principios de los sesenta —en Italia, España y luego Francia fundamentalmente- que luego no tuvo continuidad y hoy, por tanto, permanece como uno de esos objetos de estudio en que la complejidad de las relaciones intermediales de cine y literatura, encierran no pocas lecciones valiosas para la producción actual de narrativas transmediales. Se trata,

\footnotetext{
${ }^{5}$ Ley de Memoria Histórica de España (52/2007, 26 diciembre).
} 
insistimos, de un ensayo de arqueología intermedial, un enfoque que, como señala Freeman, «seeks to reconsider historical media so to illuminate, disrupt and challenge our understandings of the present» (2017: 9). De este modo, tras una definición y contextualización del cineromanzo como modalidad de «cine narrado» en formato impreso e intermedial —estamos ante un caso de lo que William J. T. Mitchell llama «nesting» o inclusión de «un medio como contenido de otro», en este caso del cinenovela dentro del formato de la fotonovela (Mitchell, 2005: 401)—, el artículo ofrece en su segunda parte un close reading de la adaptación al cineromanzo (La finestra sul cortile, 1955) del clásico del suspense fílmico Rear Window, de Alfred Hitchcock (1954). Como síntoma de la citada complejidad en la transmedialización ${ }^{6}$ desde la pantalla hasta la página impresa de un objeto cultural radicalmente popular - incluso pulp - la hipertrofia del principio de la fidelidad en la práctica de la adaptación que caracteriza al medio del cineromanzo se ve paradójicamente subvertido por la creatividad del layout o disposición editorial del diseño de las páginas de la revista, con su selección y disposición de los fotogramas de la película, que muestran cómo la experiencia narrativa del «lector» de este medio difunto no puede ser la misma, lógicamente, que la del espectador del film.

Hasta aquí estas páginas de presentación del dossier, con el agradecimiento a la dirección de la Revista Tropelías por la invitación a coordinarlo y la llamada a sus lectores a continuar el diálogo aprovechando las herramientas tecnológicas que la cibercultura misma nos proporciona ${ }^{7}$.

\section{Obras citadas}

AlBerich-PASCUAL, J. (2002): «En tránsito 7.0: Apuntes para una estética de los entornos digitales», Artnodes. Journal on Art, Science \& Technology, 2. DOI: http://doi.org/10.7238/a.v0i2.686 (última consulta, 14/07/2017).

BaEtens, Jan - VAn Looy, Jan (2003): Close Reading New Media. Analyzing Electronic Literature. Leuven, Leuven University Press.

FreEMAN, Matthew (2017): Historicising Transmedia Storytelling. Nueva York y Londres, Routledge. GIFREU, Arnau (2011): «El documental interactivo como discurso de la no ficción. Por una propuesta de definición y caracterización del nuevo género emergente», Hypertext.net, 9, en http://www. upf.edu/hipertextnet/numero-9/documental-multimedia.html (última consulta, 14/07/2017).

HERNÁNDEZ RUIZ, Javier (2015): «Plot 28, un pionero universo transmedia que radiografía el poder alienante del capitalismo de escaparate en España», Espéculo, 54 (Monográfico «Narrar en la era digital»), pp. 221-223.

JENKINS, Henry (2009): «The Revenge of the Origami Unicorn: Seven Principles of Transmedia Storytelling», en Confessions of an Aca-Fan (The Official Weblog of Henry Jenkins), 12 de

\footnotetext{
${ }^{6}$ Hablamos aquí en el sentido amplio o histórico de transmedialidad que distinguíamos en Tropelías (SánchezMesa/Baetens 2017).

7 Nar-Trans tiene sus perfiles en facebook «Congreso Internacional Narrativas Transmediales» y Twitter @Transmedia_UGR
} 
diciembre; en http://henryjenkins.org/2009/12/the_revenge_of_the_origami_uni.html_última consulta, 14/07/2017). (2017): «Adaptation, Extension, Transmedia», Literature/Film Quaterly, 45/2 (Spring).

Mitchell, William J. T. (2005): «There Are No Visual Media», Journal of Visual Culture, 2/4, pp. 395-496.

PrATten, R. (2015): Getting Started in Transmedia Storytelling. A practical Guide for Beginners. $2^{\mathrm{a}}$ edición ( $1^{\mathrm{a}}$ ed. online en http://www.tstoryteller.com/getting-started-in-transmedia-storytelling; última consulta, 14/07/2017).

RoSENDO SÁNCHEZ, Nieves (2016): «Narrativas Transmediales centradas en los personajes. Sherlock Holmes en el siglo XXI», Artnodes. Revista de Artes, Ciencia y Tecnología, 18. DOI: http://doi.org/10.7238/a.v0i18.3046 (última consulta 14/07/2017).

RYAN, Marie-Laure (2016): «Transmedia Narratology and Transmedia Storytelling», Artnodes, 18. DOI: http://doi.org/10.7238/a.v0i18.3049 (última consulta 14/07/2017).

SÁnchez-Mesa, D. - Alberich-PAscual, J. - Rosendo, N., coords. (2016): «Narrativas Transmediales», Artnodes. Revista de Artes, Ciencia y Tecnología, 18; en https://artnodes.uoc.edu/198/ volume/0/issue/18/ (última consulta 14/07/2017).

SÁnchez-Mesa, D. - AArseth, E. - Pratten, R. - Scolari, C. A. (2016): «Transmedia (Storytelling?): A polyphonic critical review», Artnodes. Revista de Artes, Ciencia y Tecnología, 18. DOI: http://doi.org/10.7238/a.v0i18.3064 (última consulta 14/07/2017).

SÁNCHEZ-MESA, D. - BAETENS, J. (2017): «La literatura en expansión. Intermedialidad y transmedialidad en el cruce entre la literatura comparada, los estudios culturales y los new media studies», Tropelías. Revista de Teoría de la Literatura y Literatura Comparada, 27, pp. 6-27; en https://papiro.unizar.es/ojs/index.php/tropelias/article/view/1536 (última consulta, 14/07/2017).

Scolari, Carlos A. - Bertetti, Paolo - Freeman, Mathew (2014): Transmedia Archeology. Storytelling in the Borderlines of Science Fiction, Comics and Pulp Magazines. Palgrave, McMillan. 